\title{
Determination of entangled quantum states of a trapped atom
}

\author{
S. Wallentowitz, R.L. de Matos Filho, and W. Vogel \\ Arbeitsgruppe Quantenoptik, Fachbereich Physik, Universität Rostock \\ Universitätsplatz 3, D-18051 Rostock, Germany
}

(September 18, 1996)

\begin{abstract}
We propose a method for measuring entangled vibronic quantum states of a trapped atom. It is based on the nonlinear dynamics of the system that appears by resonantly driving a weak electronic transition. The proposed technique allows the direct sampling of a Wigner-function matrix, displaying all knowable information on the quantum correlations of the motional and electronic degrees of freedom of the atom. It opens novel possibilities for testing fundamental predictions of the quantum theory concerning interaction phenomena.
\end{abstract}

PACS numbers: 03.65.Bz, 42.50.Vk, 32.80.Pj

\section{INTRODUCTION}

Entanglement is one of the most striking aspects of quantum mechanics [1,2]. In classical physics, two interacting systems retain their individuality during the interaction process and become completely independent of each other after their coupling has been switched off. By way of contrast, quantum theory predicts a completely different behaviour of interacting systems. When two quantum systems are brought to interact, their identities become in the course of time more and more entangled, so that a state-vector description of each system is in general precluded. They build an entangled composite system, whose state-vector cannot be separated into a product of the states of the subsystems. More surprising, the entanglement is preserved even after switching off the interaction, so that a measurement on one system will affect the other one. The individual systems can be characterised by (reduced) density matrices. However, even when the reduced density matrices of both subsystems are known, important information on the physics of their interaction and the related entanglement is lost.

Recently, entangled quantum states of an electronic system and a harmonic oscillator have been realized with trapped atoms [3] and in cavity QED [A]. The signatures of the entanglement have been partially demonstrated by quantum measurements. The determination of the full quantum statistical information of such quantum states, however, requires new types of quantum measurements. Moreover, it is interesting in this context that with both types of systems the Jaynes-Cummings model 5 and its nonlinear trapped-atom counterpart [6] could be realized [7. 8|. In the Jaynes-Cummings interaction the quantum correlation between the interacting systems has been predicted to show surprising features [9]. In all these cases methods for determining the full information on the quantum state of entangled systems are desired for getting more insight into interaction phenomena in the quantum world.

Several approaches have been proposed for determining the complete information on the quantum state of single quantum systems. In particular, homodyne tomography has been established for optical fields [10]. Further simplifications of the method have been introduced, including the direct statistical sampling of the density matrix in field-strength (quadrature) representation 11] 13], photon-number representation [14], and symplectic tomography 15]. Tomographic methods have also been realized for molecular vibrations [16] and proposed for reconstructing the motional quantum state of trapped atoms [17]. Alternatively, phase-space distributions can be determined by measuring the number statistics of the quantum state of interest, after introducing appropriate coherent displacements [18]. A method of the latter type has recently been used to reconstruct the motional state of a trapped atom [19]. For quantum systems undergoing a Jaynes-Cummings type dynamics 50 , such as high- $Q$ cavity fields [20,8] or trapped atoms [21, 6, ,7], various methods have been considered to study the quantum statistics of the bosonic subsystem (the field or the center-of-mass motion), cf. 222 24, 19]. All these methods, however, give no insight into the entanglement of quantum systems.

In this contribution we propose a method for determining entangled vibronic quantum states of a trapped atom. In Sec. II of this paper we introduce a Wigner-function matrix, which contains the full information on the composite system under study. After a brief discussion of its properties we show that it can be determined via measurements of entangled, motional number statistics. In the following section we present a concrete scheme for measuring those quantities. It relies on the nonlinearities appearing in the motional dynamics of the trapped atom interacting with laser light, which have been predicted for atomic localisations beyond the Lamb-Dicke regime [6] and confirmed in recent experiments [7]. By monitoring the electronic dynamics, combined with coherent displacements of the motional subsystem, one can perform a direct sampling of the Wigner-function matrix of the vibronic state. In Sec. IV we present a numerical simulation of the reconstruction of the Wigner-function matrix and briefly discuss the practical aspects of our method. A summary and some conclusions are given in Sec. V. 


\section{CHARACTERISATION OF ENTANGLED QUANTUM STATES}

The motional quantum state of a trapped atom is usually described by a density operator $\hat{\rho}$, which is an operator in the Hilbert space $\mathcal{H}_{\mathrm{cm}}$ of the motion of the atom in the trap. To get a phase-space description of the motional state of the atom one is lead to quasiprobability distributions such as for example the Wigner function. This function can be obtained from the density operator as follows 25, 13

$$
W(\alpha)=\langle\hat{\delta}(\alpha-\hat{a})\rangle=\operatorname{Tr}[\hat{\rho} \hat{\delta}(\alpha-\hat{a})],
$$

where the operator-valued delta-function $\hat{\delta}(\alpha-\hat{a})$ is the Fourier-transform of the displacement operator $\hat{D}(\alpha)=$ $\exp \left(\alpha \hat{a}^{\dagger}-\alpha^{*} \hat{a}\right)$ and reads as 25.

$$
\begin{aligned}
\hat{\delta}(\alpha-\hat{a}) & =\frac{1}{\pi} \int d^{2} \xi \hat{D}(\xi) e^{\alpha \xi^{*}-\alpha^{*} \xi} \\
& =\frac{2}{\pi} \hat{D}(\alpha)(-1)^{\hat{a}^{\dagger} \hat{a}} \hat{D}^{\dagger}(\alpha) .
\end{aligned}
$$

Equation (11) can be inverted to get the density operator out of the Wigner function

$$
\hat{\rho}=\int d^{2} \alpha W(\alpha) \hat{\delta}(\alpha-\hat{a})
$$

so that the knowledge of $W(\alpha)$ is equivalent to the knowledge of the motional density operator $\hat{\rho}$.

\section{A. Wigner-function matrix}

Even though the Wigner-function $W(\alpha)$ displays all the obtainable knowledge about the motional state of the atom, it gives no information on its electronic degrees of freedom. For a complete description of the atomic state - i.e. including the electronic degrees of freedom - one has to generalise the concepts leading to the definition of the Wigner-function. For this purpose we introduce the density operator $\hat{\varrho}$ of the whole system, which is now an operator in the product-space of motional and electronic Hilbert spaces $\mathcal{H}_{\mathrm{cm}} \otimes \mathcal{H}_{\mathrm{el}}$. The reduced density operators for the motional and electronic subsystems can be obtained by taking the trace over the Hilbert spaces of the electronic system (el) and the center-of-mass motion (cm), respectively,

$$
\hat{\rho}=\operatorname{Tr}_{\mathrm{el}}[\hat{\varrho}], \quad \hat{\sigma}=\operatorname{Tr}_{\mathrm{cm}}[\hat{\varrho}] .
$$

We now define a Wigner-function matrix describing the complete quantum state of the trapped atom by generalising Eq. (11),

$$
W_{i j}(\alpha)=\left\langle\hat{\delta}_{i j}(\alpha-\hat{a})\right\rangle=\operatorname{Tr}\left[\hat{\varrho} \hat{\delta}_{i j}(\alpha-\hat{a})\right]
$$

where $\hat{\delta}_{i j}(\alpha-\hat{a})$ is now an operator in the product-space $\mathcal{H}_{\mathrm{cm}} \otimes \mathcal{H}_{\mathrm{el}}$ and is defined by

$$
\hat{\delta}_{i j}(\alpha-\hat{a})=\hat{A}_{j i} \hat{\delta}(\alpha-\hat{a})
$$

Here we have used the electronic flip-operators $\hat{A}_{j i}=$ $|j\rangle\langle i|$, which describe transitions from the electronic state $|i\rangle$ to the state $|j\rangle$. In close analogy with the case of the motional sub-system (3), the density operator of the composite system can be obtained from this Wigner-function matrix as

$$
\hat{\varrho}=\sum_{i j} \int d^{2} \alpha W_{i j}(\alpha) \hat{\delta}_{j i}(\alpha-\hat{a}),
$$

showing that the complete information on the quantum state of the atom is contained in the Wigner-function matrix $W_{i j}(\alpha)$.

\section{B. Properties of the Wigner-function matrix}

Let us now discuss some properties of the Wignerfunction matrix $W_{i j}(\alpha)$. It is easy to see that its diagonal elements $W_{i i}(\alpha)$ represent the Wigner functions of the conditioned density operators $\hat{\varrho}_{i i}$ correlated to the electronic state $|i\rangle$. More precisely, this means that if one would measure the electronic state of the trapped atom to be in state $|i\rangle$, the corresponding (unnormalised) conditioned Wigner function of the motional sub-system would be $W_{i i}(\alpha)$. The norm of this conditioned Wigner function is simply the occupation probability $\sigma_{i i}$ of the electronic state $|i\rangle$, whereas the norm of the off-diagonal elements $W_{i j}(\alpha)(i \neq j)$ represents the electronic coherence

$$
\int d^{2} \alpha W_{i j}(\alpha)=\operatorname{Tr}\left[\hat{A}_{j i} \hat{\varrho}\right]=\sigma_{i j}
$$

Moreover, from Eqs. (5) and (6) it is seen that the Wigner-function matrix is hermitian

$$
W_{i j}(\alpha)=W_{j i}^{*}(\alpha)
$$

so that in order to have the full information on the quantum state under consideration, it is sufficient to know the real-valued diagonal elements of the Wigner-function matrix $W_{i i}(\alpha)$ and real and imaginary part of the offdiagonal elements $W_{i j}(\alpha)(i<j)$.

If one measures the electronic state to be in an arbitrary superposition

$$
|\psi\rangle=\sum_{i} \psi_{i}|i\rangle
$$

the conditioned density matrix of the motional subsystem is

$$
\hat{\rho}^{(|\psi\rangle)}=\operatorname{Tr}_{\mathrm{el}}[|\psi\rangle\langle\psi| \hat{\varrho}]=\sum_{i j} \psi_{i}^{*} \psi_{j} \hat{\varrho}_{i j} .
$$

The Wigner function of this motional quantum state can now be represented in terms of the Wigner-function ma$\operatorname{trix}(5)$ 


$$
W^{(|\psi\rangle)}(\alpha)=\sum_{i j} \psi_{i}^{*} \psi_{j} W_{i j}(\alpha) .
$$

This result shows that the off-diagonal elements of the Wigner-function matrix, which are in general complexvalued, contain the information on the electronic coherences and the corresponding motional states, which are entangled with these coherences.

On the other hand, if one has no information about the electronic state of the atom, the density operator of the motional sub-system is given by

$$
\hat{\rho}=\operatorname{Tr}_{\mathrm{el}}[\hat{\varrho}]=\sum_{i} \hat{\varrho}_{i i}
$$

The Wigner function of this reduced density operator reads as

$$
W(\alpha)=\sum_{i} W_{i i}(\alpha)
$$

and is simply the trace of the Wigner-function matrix.

In Figure 1 we show an example for the Wignerfunction matrix of an entangled state corresponding to the so called "Schrödinger-cat" states. The quantum state considered here has been recently prepared experimentally with a trapped atom by Monroe et al. [3]. It is a quantum superposition of two motional coherent states, $| \pm \beta\rangle$, of amplitudes $\beta$ and $-\beta$, entangled with the upper and lower electronic states, respectively. This state can be given by the following expression

$$
|\psi\rangle=\frac{1}{\sqrt{2}}(|\beta\rangle|2\rangle-|-\beta\rangle|1\rangle)
$$

The Wigner-function matrix for this entangled quantum state can be explicitely given by

$$
\begin{aligned}
& W_{11}(\alpha)=\frac{2}{\pi} \exp \left(-2|\alpha+\beta|^{2}\right) \\
& W_{22}(\alpha)=\frac{2}{\pi} \exp \left(-2|\alpha-\beta|^{2}\right) \\
& W_{12}(\alpha)=-\frac{1}{\pi} \exp \left(-2|\alpha|^{2}\right) \exp \left[2 i \operatorname{Im}\left(\alpha \beta^{*}\right)\right] .
\end{aligned}
$$

It is clearly seen, that $W_{11}(\alpha)$ and $W_{22}(\alpha)$ represent the coherent states with amplitudes $-\beta$ and $\beta$, respectively, whereas $W_{12}(\alpha)$ contains the information on the electronic coherence and the quantum interference effects inherent in the entangled state under study, see Fig. 1.

If the quantum state under consideration can be written as a product state

$$
\hat{\varrho}=\hat{\rho} \otimes \hat{\sigma},
$$

the corresponding Wigner-function matrix reads as

$$
W_{i j}(\alpha)=\sigma_{i j} W(\alpha) .
$$

States of this type contain no entanglement between the motional and electronic degrees of freedom. The corresponding Wigner-function matrix $W_{i j}(\alpha)$ is of the same shape for all indices $i, j$, determined by the motional Wigner function $W(\alpha)$ and weighted by the electronic density matrix elements $\sigma_{i j}$.
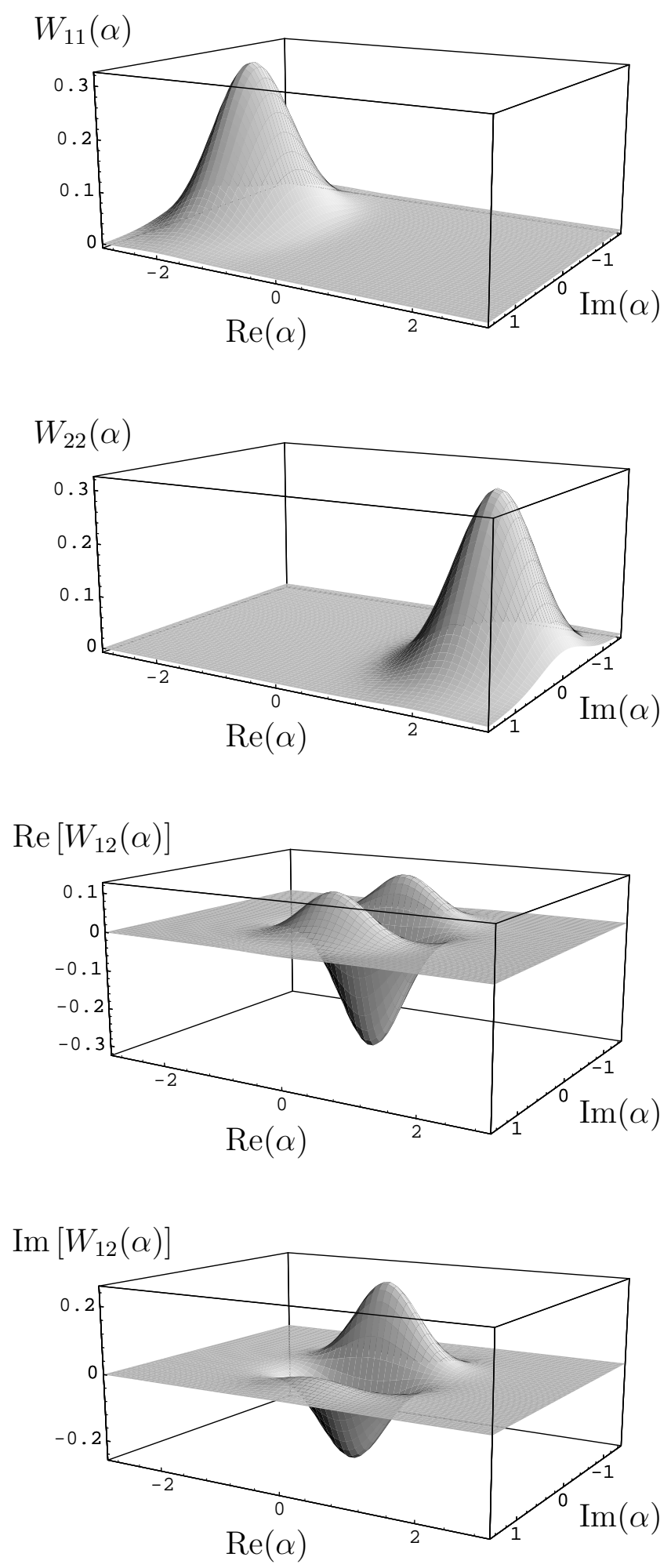

FIG. 1. Wigner-function matrix $W_{i j}(\alpha)$ of the entangled vibronic quantum state $|\psi\rangle$ [cf. Eq. (15)] with $\beta=2$. 


\section{Representation by displaced number statistics}

For determining the Wigner-function matrix $W_{i j}(\alpha)$ from measured data we note that it can be represented in terms of matrix elements of the coherently displaced density operator $\hat{\varrho}$, where only the motional diagonal elements are needed. To show this we make use of Eqs. (5), (6) together with Eq. (2). By taking the trace over the electronic sub-system, the Wigner-function matrix can be written as

$$
W_{i j}(\alpha)=\frac{2}{\pi} \operatorname{Tr}\left[\hat{\varrho}_{i j} \hat{D}(\alpha)(-1)^{\hat{a}^{\dagger} \hat{a}} \hat{D}^{\dagger}(\alpha)\right],
$$

where $\hat{\varrho}_{i j}=\langle i|\hat{\varrho}| j\rangle$ is still an operator acting on $\mathcal{H}_{\mathrm{cm}}$. By using the cyclic property of the trace and performing the trace in terms of number-states of the harmonic vibration in the trap, the Wigner-function matrix reads as

$$
W_{i j}(\alpha)=\frac{2}{\pi} \sum_{n=0}^{\infty}(-1)^{n} \varrho_{i j}^{n n}(\alpha) .
$$

Here we have made use of the coherently displaced density operator

$$
\hat{\varrho}(\alpha)=\hat{D}^{\dagger}(\alpha) \hat{\varrho} \hat{D}(\alpha) .
$$

For representing the Wigner-function matrix we only need the diagonal elements of $\hat{\varrho}(\alpha)$ with respect to the motional degree of freedom

$$
\varrho_{i j}^{n n}(\alpha)=\langle n|\langle i|\varrho(\alpha)| j\rangle| n\rangle .
$$

For convenience we will call these quantities displaced, entangled number statistics.

To get the full information on the entangled quantum state, it is sufficient to measure the displaced, entangled number statistics (22), in generalisation of the method presented in Ref. [18, 19. The initial coherent displacement can be realized by applying a radio-frequency field, as has been done in the experimental determination of the quantum state of the motional subsystem [19]. In the following we present a measurement scheme for determining the displaced, entangled number statistics $\varrho_{i j}^{n n}(\alpha)$.

\section{MEASUREMENT SCHEME}

The basic scheme consists in a three-level electronic system of $V$-type which is superimposed by the energy levels of the motion of the atom in the harmonic trap potential, see Fig. 2. A weak electronic transition $|1\rangle \leftrightarrow|2\rangle$ is the one of interest with respect to its entanglement with the motional degree of freedom. The dynamics of this transition is monitored with very high quantum efficiency by testing a strong, auxiliary transition $|1\rangle \leftrightarrow|3\rangle$ for the appearance of resonance fluorescence [26]. We assume that the weak transition is driven in the resolved sideband limit. When the laser is tuned on resonance with the vibrationless line, the corresponding interaction Hamiltonian (in the interaction picture) reads as [6,27]

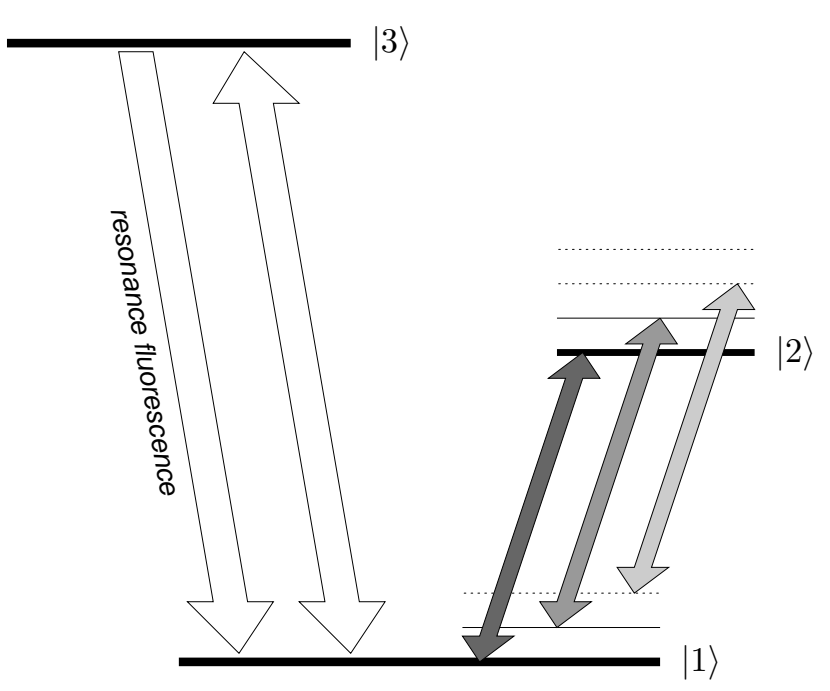

FIG. 2. Three-level electronic system of the trapped atom for the measurement of the displaced, entangled number statistics. The weak electronic transition $|1\rangle \leftrightarrow|2\rangle$ is tested by probing the strong transition $|1\rangle \leftrightarrow|3\rangle$ for resonance fluorescence.

$$
\hat{H}_{\mathrm{int}}=\frac{1}{2} \hbar \Omega \hat{f}\left(\hat{a}^{\dagger} \hat{a}\right) \hat{A}_{12}+\text { h.c. }
$$

Here $\Omega=|\Omega| \exp (i \varphi)$ is the Rabi frequency of the laser interacting with the weak electronic transition and $\varphi$ is the phase of the laser field [28]. The operators $\hat{a}$ and $\hat{a}^{\dagger}$ are the annihilation and creation operators of a vibrational quantum, respectively. The function $\hat{f}\left(\hat{a}^{\dagger} \hat{a}\right)$, describing the nonlinearities in the vibronic coupling, is given in its normally ordered form by

$$
\hat{f}\left(\hat{a}^{\dagger} \hat{a}\right)=e^{-\eta^{2} / 2} \sum_{k=0}^{\infty} \frac{(-1)^{k} \eta^{2 k}}{(k !)^{2}} \hat{a}^{\dagger k} \hat{a}^{k}
$$

with $\eta$ being the Lamb-Dicke parameter, characterising the localisation of the atom in the trap. The interaction Hamiltonian (23) fulfils the condition $\left[\hat{n}, \hat{H}_{\text {int }}\right]=0$ of back-action evasion for the vibrational number operator $\hat{n}=\hat{a}^{\dagger} \hat{a}$, so that the latter is a constant of motion. Consequently, this interaction couples only between vibronic density matrix elements $\varrho_{i j}^{m n}$ having the same motional indices $(m, n)$. This renders it possible to determine the entangled motional number statistics $\varrho_{i j}^{n n}$ of the initial vibronic state by monitoring the dynamics of the electronic transition $|1\rangle \leftrightarrow|2\rangle$.

One possibility to obtain the matrix elements $\varrho_{i j}^{n n}(\alpha)$ is the measurement of the time dependence of the occupation of state $|2\rangle$. This dynamics is completely determined by those matrix elements. However, their reconstruction requires some effort of data analysing by Fourier or other appropriate techniques, as considered for the Jaynes-Cummings dynamics [22, 23, 19, 8].

In the following we will deal with an alternative method that directly yields the quantities desired in Eq. (20). It is related to the scheme proposed in Ref. 27] 
for the quantum nondemolition measurement of the motional energy of a trapped atom. After a well controlled interaction time of the laser resonant to the weak electronic transition, a laser pulse is applied on the strong transition in order to probe for the appearance of resonance fluorescence. The occurrence of fluorescence detects the atom in the electronic ground state $|1\rangle$ and its absence in the excited electronic state $|2\rangle$. We will focus our attention to the no-fluorescence events, since they preclude any disturbance of the motional state via light scattering by the strong transition. Alternating sequences of light pulses on the weak and the strong transition allow to reduce the motional state to a Fock state, which is predetermined by time control of the pulse sequence on the weak transition. Eventually, the desired information on the entangled number statistics $\varrho_{i j}^{n n}(\alpha)$ is directly given by the probability of realizing such a sequence of interaction-probe cycles, for each predetermined Fock state $|n\rangle$.

Assume that, subsequent to the coherent displacement of the motional subsystem, the atom is probed for fluorescence on the strong transition after an interaction time $\tau$ of the laser with the weak transition. Provided the atom is detected in the electronic state $|2\rangle$ (no fluorescence), the density operator of the system reduces to the (unnormalised) operator

$$
\hat{\varrho}^{(\mathrm{red})}(\tau)=|2\rangle\langle 2| \otimes \hat{\rho}^{(\mathrm{red})}(\tau),
$$

where $\hat{\rho}^{(\mathrm{red})}(\tau)=\langle 2|\hat{\varrho}(\tau)| 2\rangle$ is the corresponding (unnormalised) density operator of the motional subsystem related to the electronic state $|2\rangle$. In view of the interaction Hamiltonian (23), the diagonal elements of $\hat{\rho}^{(\mathrm{red})}(\tau)$ in number-state representation are given by

$$
\begin{aligned}
\rho_{n n}^{(\mathrm{red})}(\tau)= & \varrho_{22}^{n n}(\alpha) \cos ^{2}\left(\frac{\Omega_{n} \tau}{2}\right)+\varrho_{11}^{n n}(\alpha) \sin ^{2}\left(\frac{\Omega_{n} \tau}{2}\right) \\
& +\operatorname{Im}\left[\varrho_{12}^{n n}(\alpha) e^{-i \varphi}\right] \sin \left(\Omega_{n} \tau\right),
\end{aligned}
$$

where $\Omega_{n}=|\Omega| L_{n}\left(\eta^{2}\right) \exp \left(-\eta^{2} / 2\right)$ are the nonlinear vibronic Rabi frequencies, $L_{n}(x)$ being Laguerre polynomials.

Let us consider the effect of $k$ of these interactionprobe cycles with interaction times $\tau_{1}, \ldots, \tau_{k}$, each one accompanied by no fluorescence [29]. The resulting (unnormalised) motional number statistics is conditioned on the times $\tau_{1}, \ldots, \tau_{k}$, at which the reductions to the state $|2\rangle$ occurred, and reads as

$$
\rho_{n n}^{(\mathrm{red})}\left(\tau_{k}, \ldots, \tau_{1}\right)=\prod_{q=2}^{k} \cos ^{2}\left(\frac{\Omega_{n} \tau_{q}}{2}\right) \rho_{n n}^{(\mathrm{red})}\left(\tau_{1}\right) .
$$

The probability $P\left(\tau_{k}, \ldots, \tau_{1}\right)$ to obtain such a sequence of interaction-probe cycles is given by the trace of $\hat{\rho}^{\text {(red) }}\left(\tau_{k}, \ldots, \tau_{1}\right)$,

$$
P\left(\tau_{k}, \ldots, \tau_{1}\right)=\sum_{n=0}^{\infty} \rho_{n n}^{(\mathrm{red})}\left(\tau_{k}, \ldots, \tau_{1}\right),
$$

and can be experimentally determined by repeating this procedure many times and counting the number of times one was successful in obtaining such a sequence.

An adequate choice of the interaction times $\tau_{k}$ allows to map $\rho_{n n}^{(\text {red })}\left(\tau_{1}\right)$ onto the probability $P\left(\tau_{k}, \ldots, \tau_{1}\right)$. For mapping the particular element $\rho_{m m}^{(\text {red })}\left(\tau_{1}\right)$, it is useful to choose the interaction times $\tau_{2}, \ldots, \tau_{k}$ as

$$
\tau_{2}, \ldots, \tau_{k}=\frac{2 \pi}{\Omega_{m}} p, \quad p=1,2, \ldots,
$$

where $p$ can be set to a different integer at each interaction cycle. From Eq. (27) it is seen that after an appropriate number $k \geq k_{\text {min }}$ of interaction-probe cycles $\rho_{n n}^{(\text {red })}\left(\tau_{k}, \ldots, \tau_{1}\right)$ reduces to

$$
\rho_{n n}^{(\text {red })}\left(\tau_{k}, \ldots, \tau_{1}\right)=\delta_{n m} \rho_{m m}^{(\text {red })}\left(\tau_{1}\right) .
$$

The other matrix elements $(n \neq m)$ are suppressed by the product of cosines in Eq. (27), provided that different motional number states are efficiently discriminated by the vibronic Rabi frequencies $\Omega_{n}$ [27]. It is easy to see [cf. Eqs (28), (30)] that in this case the probability $P\left(\tau_{k}, \ldots, \tau_{1}\right)$ reflects the quantity $\rho_{m m}^{(\text {red })}\left(\tau_{1}\right)$ and that further interaction-probe cycles will not change anything. Since the interaction times $\tau_{k}$ are specified from the beginning, the minimum number $k_{\text {min }}$ of cycles needed to complete the mapping process can be evaluated.

The number statistics $\rho_{m m}^{(\mathrm{red})}\left(\tau_{1}\right)$ obtained in this manner can be used to determine the entangled, motional number statistics $\varrho_{i j}^{m m}(\alpha)$ of the displaced initial vibronic state via Eq. (26). For this purpose, three different choices of the first interaction time $\tau_{1}$ are desired:

(i) $\tau_{1}=0$ : After detecting the atom in the excited electronic state $|2\rangle$ (no detection of fluorescence), $\rho_{m m}^{(\mathrm{red})}\left(\tau_{1}\right)$ is given by

$$
\rho_{m m}^{(\mathrm{red})}\left(\tau_{1}\right)=\varrho_{22}^{m m}(\alpha)
$$

(ii) $\tau_{1}=\pi / \Omega_{m}$ : For this choice the diagonal elements of the motional density matrix are given by

$$
\rho_{m m}^{(\mathrm{red})}\left(\tau_{1}\right)=\varrho_{11}^{m m}(\alpha) .
$$

(iii) $\tau_{1}=\pi /\left(2 \Omega_{m}\right)$ : In this case one arrives at

$$
\begin{aligned}
\rho_{m m}^{(\mathrm{red})}\left(\tau_{1}\right)= & \frac{1}{2}\left[\varrho_{11}^{m m}(\alpha)+\varrho_{22}^{m m}(\alpha)\right] \\
& +\operatorname{Im}\left[\varrho_{12}^{m m}(\alpha) e^{-i \varphi}\right] .
\end{aligned}
$$

Choosing two laser phases, $\varphi=0$ and $\varphi=-\pi / 2$, one can obtain the imaginary and real part of $\varrho_{12}^{m m}(\alpha)$, respectively, by subtracting one half of the outcomes from (i) and (ii).

Eventually, any positive event (series of no fluorescence) recorded in this manner can be stored in the computer for a direct sampling of the Wigner-function matrix (to be normalised by the number of trials) according to Eq. (20). 


\section{NUMERICAL SIMULATIONS}

A typical example for an entangled vibronic quantum state of a trapped atom is the state given in Eq. (15). In Fig. 3 we show a simulation of all steps of the scheme for the determination of the Wigner-function matrix of the state $|\psi\rangle$ according to the method proposed above. The maximum pulse length of the laser resonant to the weak transition is of the order of $6 \mu$ s for a typical Rabi frequency of $\Omega / 2 \pi=500 \mathrm{kHz}$ [30]. For interaction times of this order the electronic relaxation of the weak transition is negligible. The time needed for the data acquisition of a point in phase space is approximately 30 seconds, so that the complete sampling of one matrix element of the Wigner-function matrix $W_{i j}(\alpha)$ as shown in Fig. 3 would take about 3 hours. This is comparable with the data-acquisition time needed for the reconstruction of the quantum state of the motional subsystem, by measurement of the time evolution of the electronic inversion 19. The advantage of our scheme, however, consists in the fact that the desired number statistics is directly observed, with no need for inverting systems of linear equations with noisy data input.

The diagonal elements $W_{22}(\alpha)$ and $W_{11}(\alpha)$ simply represent the Wigner functions of the coherent states $|\beta\rangle$ and $|-\beta\rangle$ respectively. The off-diagonal elements $W_{i j}(\alpha)$ contain the more interesting information on quantum interferences inherent in the entangled state (15). Comparing with the theoretical result of $W_{i j}(\alpha)$ given in Fig. 1, a good agreement between theory and simulation is found. Note that the entanglement is reflected by the fact that the Wigner-function matrix is different for different electronic indices. If one would measure the Wigner function of the motional sub-system, $W(\alpha)=\sum_{i} W_{i i}(\alpha)$, the result would be nothing but an incoherent superposition of two coherent states, completely concealing the nonclassical character of the atomic state. That is, in the chosen example the nonclassical nature of the system is manifested by the entanglement. Our measurement scheme is directly suited to demonstrate these features.

\section{SUMMARY AND CONCLUSION}

In conclusion we have proposed a measurement technique for obtaining the full information on entangled vibronic states of a trapped atom. First of all, this requires a characterisation of the full quantum state of the composed system which can be related to an appropriate measurement scheme. For this purpose we define a Wigner-function matrix which has the character of a density matrix with respect to the electronic degrees of freedom, and of a Wigner function for the motional subsystem. This Wigner-function matrix is readily related to the displaced, entangled motional number-statistics of the atom.

For the determination of the Wigner-function matrix we have presented a method that allows the measurement of the displaced, entangled motional number-statistics. It
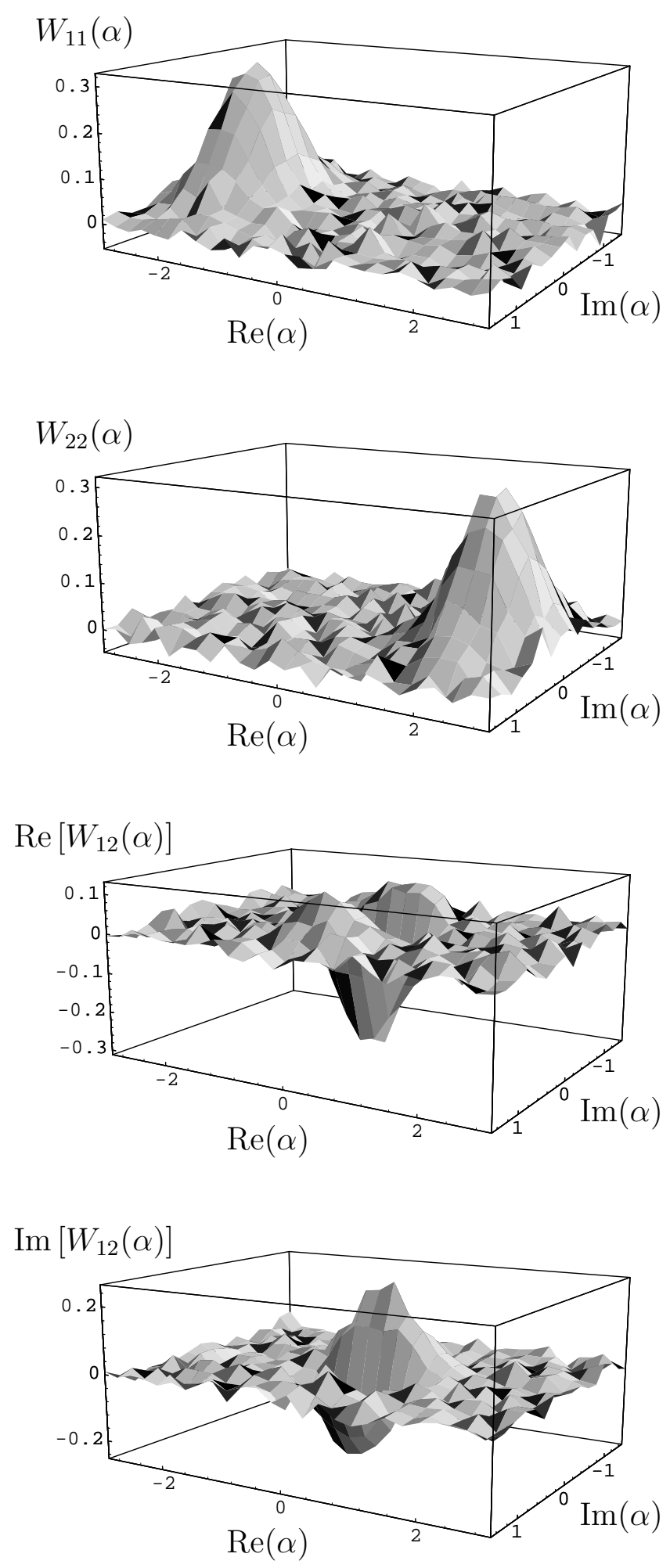

FIG. 3. Wigner-function matrix $W_{i j}(\alpha)$ of the entangled vibronic quantum state $|\psi\rangle$ [Eq. (15)] with $\beta=2$. Each matrix element $\varrho_{i j}^{m m}(0 ; \alpha)$ has been numerically sampled with 1000 trials and a sequence of $k=30$ interaction-probe cycles on a $25 \times 15$-grid. The Lamb-Dicke parameter is $\eta=0.1$. 
is based on the nonlinearities appearing in the vibronic coupling of a resonantly driven, weak electronic transition. This coupling fulfils the back-action evasion criterion for the number of motional quanta. It eventually allows, in combination with electronic-state reductions by fluorescence measurements, the direct statistical sampling of a Wigner-function matrix displaying the entanglement between the motional and the electronic degrees of freedom of the atom. The method proposed here opens new perspectives for experimental demonstrations of fundamental properties of interacting quantum systems.

This work was supported by the Deutsche Forschungsgemeinschaft.

[1] E. Schrödinger, Naturwiss. 23, 807-812; 823-828; 844-849 (1935).

[2] A. Einstein, B. Podolsky, and N. Rosen, Phys. Rev. 47, 777 (1935).

[3] C. Monroe, D.M. Meekhof, B.E. King, and D.J. Wineland, Science 272, 1131 (1996).

[4] M. Brune, E. Hagley, J. Dreyer, X. Maitre, A. Maali, C. Wunderlich, J.M. Raimond, and S. Haroche, Phys. Rev. Lett. 77, 4887 (1996).

[5] E.T. Jaynes and F.W. Cummings, Proc. IEEE 51, 89 (1963).

[6] W. Vogel and R.L. de Matos Filho, Phys. Rev. A 52, 4214 (1995). For a treatment of the modifications due to the micromotion in a Paul trap see 24].

[7] D.M. Meekhof, C. Monroe, B.E. King, W.M. Itano, and D.J. Wineland, Phys. Rev. Lett. 76, 1796 (1996).

[8] M. Brune, F. Schmidt-Kaler, A. Maali, E. Hagley, J.M. Raimond, and S. Haroche, Phys. Rev. Lett. 76, 1800 (1996).

[9] S.J.D. Phoenix and P.L. Knight, Ann. Phys. (N.Y.) 186, 381 (1988); ibid. Phys. Rev. A 44, 6023 (1991); J. GeaBanacloche, Phys. Rev. Lett. 65, 3385 (1991).

[10] D.T. Smithey, M. Beck, M.G. Raymer, and A. Faridani, Phys. Rev. Lett. 70, 1244 (1993).

[11] H. Kühn, D.-G. Welsch, and W. Vogel, J. Mod. Opt. 41, 1607 (1994).

[12] A. Zucchetti, W. Vogel, M. Tasche, and D.-G. Welsch, Phys. Rev. A 54, 1678 (1996).

[13] W. Vogel and D.-G. Welsch, Lectures on Quantum Optics (Akademie Verlag / VCH Publishers, Berlin, 1994).

[14] G.M. D'Ariano, C. Macchiavello, and M.G.A. Paris, Phys. Rev. A 50, 4298 (1994); G.M. D'Ariano, U. Leonhardt, and H. Paul, Phys. Rev. A 52, R1801 (1995).

[15] S. Mancini, V.I. Man'ko, and P. Tombesi, Quant. Semiclass. Opt. 7, 615 (1995); O.V. Man'ko, J. Russ. Laser Research 17, 439 (1996).

[16] T.J. Dunn, I.A. Walmsley, and S. Mukamel, Phys. Rev. Lett. 74, 884 (1995).

[17] S. Wallentowitz and W. Vogel, Phys. Rev. Lett. 75, 2932 (1995); Phys. Rev. A 54, in press; J.F. Poyatos, R. Walser, J.I. Cirac, and P. Zoller, Phys. Rev. A 53, R1966
(1996); C. D'Helon and G.J. Milburn, Phys. Rev. A 54, R25 (1996).

[18] S. Wallentowitz and W. Vogel, Phys. Rev. A 53, 4528 (1996). K. Banaszek and K. Wódkiewicz, Phys. Rev. Lett. 76, 4344 (1996). For the representation of the quasidistributions underlying the method see also K.E. Cahill and R.J. Glauber, Phys. Rev. 177, 1882 (1969); H. Moya-Cessa and P.L. Knight, Phys. Rev. A 48, 2479 (1993).

[19] D. Leibfried, D.M. Meekhof, B.E. King, C. Monroe, W.M. Itano, and D.J. Wineland, Phys. Rev. Lett. 77, 4281 (1996).

[20] G. Rempe, H. Walther, and N. Klein, Phys. Rev. Lett. 58, 353 (1987).

[21] C.A. Blockley, D.F. Walls and H. Risken, Europhys. Lett. 17, 509 (1992); J.I. Cirac, R. Blatt, A.S. Parkins, and P. Zoller, Phys. Rev. A 49, 1202 (1994).

[22] W. Vogel, D.-G. Welsch, and L. Leine, J. Opt. Soc. Am. B 4, 1633 (1987).

[23] P.J. Bardroff, E. Mayr, and W.P. Schleich, Phys. Rev. A 51, 4963 (1995); P.J. Bardroff, E. Mayr, W.P. Schleich, P. Domokos, M. Brune, J.M. Raimond, and S. Haroche, Phys. Rev. A 53, 2736 (1996);

[24] P.J. Bardroff, C. Leichtle, G. Schrade, and W.P. Schleich, Phys. Rev. Lett. 77, 2198 (1996).

[25] K.E. Cahill and R.J. Glauber, Phys. Rev. 177, 1882 (1969).

[26] W. Nagourney, J. Sandberg, and H.G. Dehmelt, Phys. Rev. Lett. 56, 2797 (1986); Th. Sauter, W. Neuhauser, R. Blatt, and P.E. Toschek, Phys. Rev. Lett. 57, 1696 (1986); J.C. Bergquist, R.G. Hulet, W.M. Itano, and D.J. Wineland, Phys. Rev. Lett. 57, 1699 (1986).

[27] R.L. de Matos Filho and W. Vogel, Phys. Rev. Lett. 76, 4520 (1996); L. Davidovich, M. Orszag, and N. Zagury, Phys. Rev. A 54, 5118 (1996).

[28] For a Raman-type excitation the phase $\varphi$ is the difference phase of the two Raman beams and can be held very stable.

[29] The selection of no-fluorescence trajectories has also been proposed for stochastic cooling, cf. J. Eschner, B. Appasamy, and P.E. Toschek, Phys. Rev. Lett. 74, 2435 (1995).

[30] C. Monroe, D.M. Meekhof, B.E. King, S.R. Jefferts, W.M. Itano, D.J. Wineland, and P. Gould, Phys. Rev. Lett. 75, 4011 (1995). 